\title{
QueryBlazer: Efficient Query Autocompletion Framework
}

\author{
Young Mo Kang \\ ykang@salesforce.com \\ Salesforce Research \\ Palo Alto, California
}

\author{
Wenhao Liu \\ wenhao.liu@salesforce.com \\ Salesforce Research \\ Palo Alto, California
}

\author{
Yingbo Zhou \\ yingbo.zhou@salesforce.com \\ Salesforce Research \\ Palo Alto, California
}

\begin{abstract}
Query autocompletion is an essential feature in search engines that predicts and suggests query completions to a user's incomplete prefix input, a critical feature to enhance the user experience. While a generic lookup-based system can provide completions with great efficiency, it is unable to address prefixes not seen in the past. On the other hand, a generative system can complete unseen queries with superior accuracy but requires substantial computational overhead at runtime, making it costly for a large-scale system.

Here, we present an efficient, fully-generative query autocompletion framework. Our framework employs an n-gram language model at a subword-level and exploits the n-gram model's inherent data structure to precompute completions prior to runtime. Evaluation results on public dataset show that our framework is not only as effective as previous systems with neural language models, but also reduces computational overhead at runtime, expediting the speed by more than two orders of magnitude. The goal of this work is to showcase a generative query completion system that is an attractive choice for large-scale deployments.
\end{abstract}

\section{CCS CONCEPTS}

- Information systems $\rightarrow$ Query suggestion; Language models; Probabilistic retrieval models.

\section{KEYWORDS}

query; queries; autocompletion; suggestion; subword; completion; language model; n-gram

\section{ACM Reference Format:}

Young Mo Kang, Wenhao Liu, and Yingbo Zhou. 2021. QueryBlazer: Efficient Query Autocompletion Framework. In Proceedings of the Fourteenth ACM International Conference on Web Search and Data Mining (WSDM '21), March 8-12, 2021, Virtual Event, Israel. ACM, New York, NY, USA, 9 pages. https: //doi.org/10.1145/3437963.3441725

\section{INTRODUCTION}

Query autocompletion (QAC) is a convenient feature widely used in search engines today that provides query completions from a user's incomplete input prefix ranked by a scoring function. QAC reduces user's typing on average from $25 \%$ to $50 \%$ [3, 45] and has been an essential feature to enhance the user experience. QAC application is

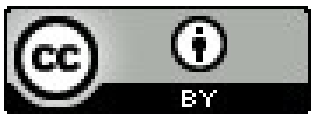

This work is licensed under a Creative Commons Attribution International 4.0 License.

WSDM '21, March 8-12, 2021, Virtual Event, Israel.

(C) 2021 Copyright held by the owner/author(s).

ACM ISBN 978-1-4503-8297-7/21/03.

https://doi.org/10.1145/3437963.3441725 not just limited to search engines and has become a part of various services in Facebook, Twitter, Amazon, and Youtube [10].

A lookup-based approach has been a traditional method to QAC. In this approach, the system stores the past query history and retrieves the queries that match the user's input prefix from the database. With optimized data structures $[12,19,26]$ the lookupbased approach is very efficient and can suggest query completions almost instantaneously in the order of microseconds [19]. Some queries however are new and unseen, making it challenging to provide accurate completions. According to Google [2], 15\% of queries submitted to its search engine have never been seen before. These unseen queries cannot be retrieved from the database and hence must rely on an alternative approach. These queries are often referred to as the long-tail queries [40].

More recently, a new paradigm in QAC has emerged to resolve the aforementioned limitation. This new approach [32] achieves superior accuracy for previously unseen queries by generating completions from a neural language model via beam search. This fullygenerative approach is very effective in suggesting accurate completions, but comes at the cost of large computational overhead at runtime, rendering it costly to scale for large search engines. Several studies [21, 42] have improved its efficiency, but the completion speed is still in the order of milliseconds, lagging behind the traditional lookup-based approach by three orders of magnitude. The large gap in the computational efficiency serves as one of the main bottlenecks for adoption of a generative system in a large scale, despite its advantage in completion accuracy.

In this paper, we focus on the prefix-match mode [22] of query completions where the system suggests strings that match the user's partial input as a prefix. Specifically, we propose a fully-generative system employing an n-gram language model at a subword-level. Our system is not only able to suggest queries from the log history but is also able to generate a new word or phrase that it has never seen before. For instance, given the user's partial input "instagra", our model trained on the AOL dataset [33] suggests new ${ }^{1}$ terms "instagram" and "instagram.com" within the top-5 completions. We exploit the n-gram model's inherent data structure and devise techniques to carry out query completions prior to runtime, eliminating substantial runtime overhead. We evaluate our system on two different public datasets and demonstrate that it outperforms previous methods in completion accuracy and at the same time achieves 100x speed-up. The main research question we investigate is:

Can we design a generative QAC system that is an attractive choice for large-scale deployments?

We release the source code to the public ${ }^{2}$.

\footnotetext{
${ }^{1}$ The word "instagram" is not present in the dataset, as Instagram was founded four years after the dataset was released.

${ }^{2}$ https://github.com/salesforce/QueryBlazer
} 


\section{RELATED WORK}

QAC systems can be roughly categorized into two broad branches: a database lookup approach and a generative approach.

Most popular completion (MPC) [8] is the standard lookup-based QAC technique that returns the most frequently observed queries from the past that match the user's input prefix. The method is typically implemented using a trie data structure, storing past queries and their counts. Given a user's query prefix, the system traverses the child nodes and retrieves the queries with the highest counts, where the past query count serves as the ranking score. Several techniques have been proposed $[12,19,26]$ for optimal runtime efficiency. One major limitation, however, is that this approach cannot suggest completions for the long-tail queries.

Generative methods, on the other hand, synthesize query completions that are not in the past log database. Szpektor et al. [40] proposed a method for generating queries using fixed rules and templates. Bhatia et al [9] and Maxwell et al. [27] detailed generative QAC systems in the absence of query logs that synthesize completions by merging the user prefix with n-grams mined from an external text corpus. Mitra and Craswell [30] generated query completions by merging rare prefixes with popular $n$-gram suffixes and ranked them using a convolutional latent semantic model [38]. These studies are orthogonal to ours, as they employ heuristic methods for generating the completions rather than a full beam-search using a language model.

The most relevant work to ours are [12, 32, 42]. In [32] Park and Chiba proposed a fully-generative system where a character-level neural language model is trained on the past query data and is used to generate completion candidates via beam search. This method achieves the state-of-the-art completion accuracy for previously unseen queries, but the runtime speed is not reported. Wang et al. [42] developed an efficient beam search algorithm suitable for a neural language model, achieving a real time completion speed in a multithreaded-CPU setting. Kim [21] investigated a neural language model at a subword-level as an alternative to the character-level granularity, leading to faster runtime while maintaining comparable accuracy to the character-level model. One major difference that separates our work from these previous studies is that our system does not perform the beam search at runtime; rather, it is carried out during the training stage. Specifically, our method allows precomputation of beam search for every possible user input. We provide in-depth discussion in Section 4.2.

\section{PRELIMINARIES}

\subsection{Subword Encoding}

Subword-level models have been highly successful in various natural language processing (NLP) tasks, ranging from machine translation [37, 41], language modeling [15, 34], and automatic speech recognition $[16,43]$. A subword-level language model combines benefits from both of the character-level and the word-level language models in that it reduces the sequence length compared to the character representation, and at the same time resolves out-ofvocabulary $(\mathrm{OOV})$ issues inherent in the word-level representation. We briefly review three subword encoding schemes that are relevant to our work.
Introduced to address rare words in machine translation, bytepair encoding (BPE) [37] merges pairs of tokens in the order of frequencies observed during the training stage. The subword vocabulary consists of all individual characters and the merged tokens. $\mathrm{BPE}$ is a deterministic segmentation method in that any given word is segmented to a unique sequence of subword tokens. In the QAC setting, BPE poses a segmentation boundary problem because the given prefix may not align with the true boundary [21].

Subword regularization (SR) [23] exploits inherent ambiguity in subword segmentation by training a model with multiple segmentation boundaries for the same vocabulary. This leads to a model regularization effect as well as data augmentation. SR alleviates the segmentation boundary problem [21] because the language model learns various representations occurring from different segmentation boundaries. This effect, however, diminishes with a larger size of the subword vocabulary, as the number of possible segmentation grows exponentially.

In our framework, we employ the longest prefix matching (LPM) algorithm, another variant of subword encoding scheme used in [1, $36,44]$. LPM is also a deterministic segmentation method in which characters are encoded into the longest matching subwords from left to right. Unlike BPE and SR where merging of characters into a subword can take place anywhere within the query, an LPM encoder merges characters only from left to right in a greedy fashion. This property yields two main advantages over the previous encoding algorithms: 1) a fewer number of potential segmentation boundaries and 2) a direct translation to a finite state transducer (FST). The former results in reduced search space, while the latter enables us to precompute all segmentation variations in advance, contributing to runtime efficiency. We discuss the details in Section 4.1.

\subsection{Finite State Transducer}

Two main components that constitute our framework are the subword encoder represented as an FST and the n-gram language model represented as a weighted FST. We therefore provide a minimal informal review on a (weighted) FST in the context of our work. For a more complete and formal review, readers are directed to [31].

A deterministic FST is a finite state machine that converts or transduces a sequence of input labels into a sequence of output labels by a series of state transitions uniquely determined by the input. An FST $G$ consists of a finite state $\operatorname{set} Q$, a finite input alphabet $\mathcal{X}$, a finite output alphabet $\mathcal{Y}$, an initial (start) state $i \in Q$, a final (exit) state set $\mathcal{F} \subseteq Q$, and a finite set of transitions (arcs) $\mathcal{T} \subseteq$ $Q \times(\mathcal{X} \cup\{\epsilon\}) \times(\mathcal{Y} \cup\{\epsilon\}) \times Q$. A transition $t \in \mathcal{T}$ is represented as a four-tuple ( $p, x, y, n)$ where $p, n \in Q$ are the states before (previous) and after (next) the transition, respectively, $x \in X \cup\{\epsilon\}$ is the input label, and $y \in \mathcal{Y} \cup\{\epsilon\}$ is the output label. The special symbol $\epsilon$ denotes a blank input/output label.

A path refers to a sequence of consecutive transitions from the initial state $i$ to the final state $f \in \mathcal{F}$. The input label sequence $\mathbf{x}$ associated with the path is the concatenation of the input labels for each transition, discarding $\epsilon$. The output label sequence $y$ is obtained in a similar manner. An FST transduces an input sequence $\mathbf{x}$ to an output sequence $\mathbf{y}$ if there exists a path associated with $\mathbf{x}, \mathbf{y}$. A deterministic FST has at most one path corresponding to any given input sequence; that is, the output sequence, if any, is uniquely determined by the input sequence. 


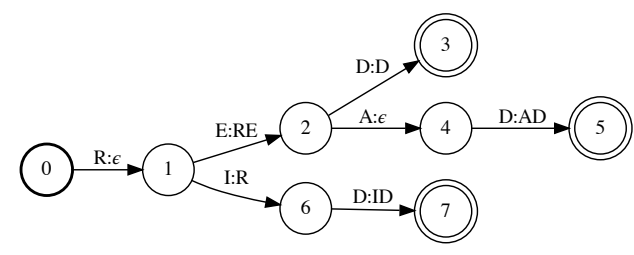

Figure 1: A toy example of a FST graph. States are represented by circles and denoted with the index. The input label $x$ and the output label $y$ of a transition are marked on the corresponding arc as $x: y$.

Figure 1 shows an example of an FST. State 0 is the initial state $i$ and is denoted by a bold circle. Each transition is depicted as a directed arrow. For example, there is a transition from state 4 to 5 with the input symbol "D" and the output symbol "AD". States 3, 5, and 7 are final states, denoted by double circles. There exists a path associated with the input character sequence "RED" and the output subword sequence "RE/D", represented by the state transitions 0 1-2-3. Similarly, the graph transduces input character sequences "READ" and "RID" into the output subword sequences "RE/AD" and "R/ID", respectively.

A weighted FST extends an FST by associating a weight semiring [25] to each transition and the final states. A path then collects a sequence of weight values from the transitions and the final state. A distance associated with the path is the product of the weight values of the path, where the product operation $\otimes$ is defined by its semiring type. We represent an n-gram language model as a deterministic weighted FST in the tropical semiring. In this case, the weight value represents the negative log likelihood, and the $\otimes$ operator equates to the arithmetic addition.

\section{FRAMEWORK}

Figure 2 illustrates a high-level overview of the proposed framework. The system consists of two main components: a subword encoder and an n-gram language model. During the training stage, we extract a subword vocabulary set from the train dataset-usually the past query history-and construct an encoder as an FST. We then train an n-gram language model at the subword-level and represent it as a weighted FST. The FST representations of these two components play an integral part in runtime optimization.

At runtime, the user's query prefix is fed into the subword encoder as a sequence of input characters. The encoder then outputs all subword sequences that can result from the partial input. Suppose that the subword vocabulary contains only three subwords that start with the letter "A", namely "A", "AN", and "AG". Also suppose that there is no subword that starts with "BA" in the vocabulary. Then, any query that begins with the prefix "BA" must begin with prefix "B/A/", "B/AG/", or "B/AN/" in its subword representation. We refer to these as the segmentation candidates. The language model then performs beam search on each of the candidates to generate top- $k$ completions. For example, given the subword prefix "B/AG/", the beam search may return "EL/" and "U/ET/TE/" as the top-2 completions. We refer to these as the completion candidates. Finally, top- $k$ completions from each segmentation are merged into a single pool, from which the final top- $k$ completions are returned to the user.

In practice, we employ techniques to substantially improve execution speed at runtime. We precompute and store the segmentation candidates from each state of the encoder. This is possible with the FST representation of the LPM encoder. A more substantial speedup comes from precomputation of beam search, storing the top- $k$ completion candidates for every $\mathrm{n}$-gram history. With these two, query completions to any user input can be efficiently computed.

\subsection{Encoder}

Let $\mathcal{X}, \mathcal{Y}$ be a set of input characters $x$ and output subwords $y$, respectively. Typically, $\mathcal{X}$ consists of normalized alphabet letters and some punctuation marks, and $\mathcal{Y}$ consists of concatenations of input characters. An FST encoder $E$ transduces a sequence of characters $\mathbf{x}=\left(x_{1}, x_{2}, \ldots, x_{m}\right)$ into a sequence of subwords $\mathbf{y}=$ $\left(y_{1}, y_{2}, \ldots, y_{n}\right)$. We assume that the subword alphabet set is the superset of the input alphabet set, i.e., $\mathcal{X} \subseteq \mathcal{Y}$.

4.1.1 Construction. In the following, we detail the construction process for the encoder module.

Stage 1 . We begin by constructing a trie data structure with the subword vocabulary as the keys. We then convert the trie into an FST as shown in Figure 3a. The root of the trie is the start state and the leaf nodes are the exit states of the FST. Each exit state is associated with a subword $y \in \mathcal{Y}$. For example, state 4 is associated with the subword "ANAN". The output of each transition is set to $\epsilon$.

Stage 2. We add a transition from each exit state to the start state with input label $\phi$ and the output label $y$ associated with the state. Following the convention in [4], $\phi$ denotes a special symbol for a failure or a fallback transition, which is taken only if there is no outgoing transition that matches the given input. We also let the root be the only exit state. Figure $3 \mathrm{~b}$ illustrates the FST after these changes. Not every input sequence can be encoded at this stage. For example, $E$ can encode an input "BANANA" into the output subwords "B/ANAN/A/", but cannot encode an input "ANN", as there is no viable transition for the last input character " $N$ " at state 2. We add fallback transitions in the following stage.

Stage 3. Algorithm 1 describes a breadth-first traversal to add a $\phi$-transition at every intermediate state. In a nutshell, the algorithm traverses all states in the encoder from the start state and adds a series of $\phi$-transitions, if it is not at the start state and does not already have a $\phi$-transition. The $\phi$-transitions allow the encoder to fall back to the next longest subword matching the input prefix. It recursively follows the fallback path from the previous state and makes one more transition matching the last character in the input. Here, we define the method $\operatorname{Transition}(\mathrm{p}, \mathrm{x})$ as follows. If the input symbol $x$ is not $\phi$, it will make a matching transition ${ }^{3}$ from the state p. If $x$ is $\phi$, it will make a single $\phi$-transition. In both cases, if a $\phi$-transition is the only outgoing arc at the destination state ${ }^{4}$, the method will repeatedly take the $\phi$-transition. Finally, the method

\footnotetext{
${ }_{3 \text { may be preceded }}$ by one or more consecutive $\phi$-transitions until the matching arc is found.

${ }^{4}$ such as state 4, 5, and 6 in Figure 3b.
} 


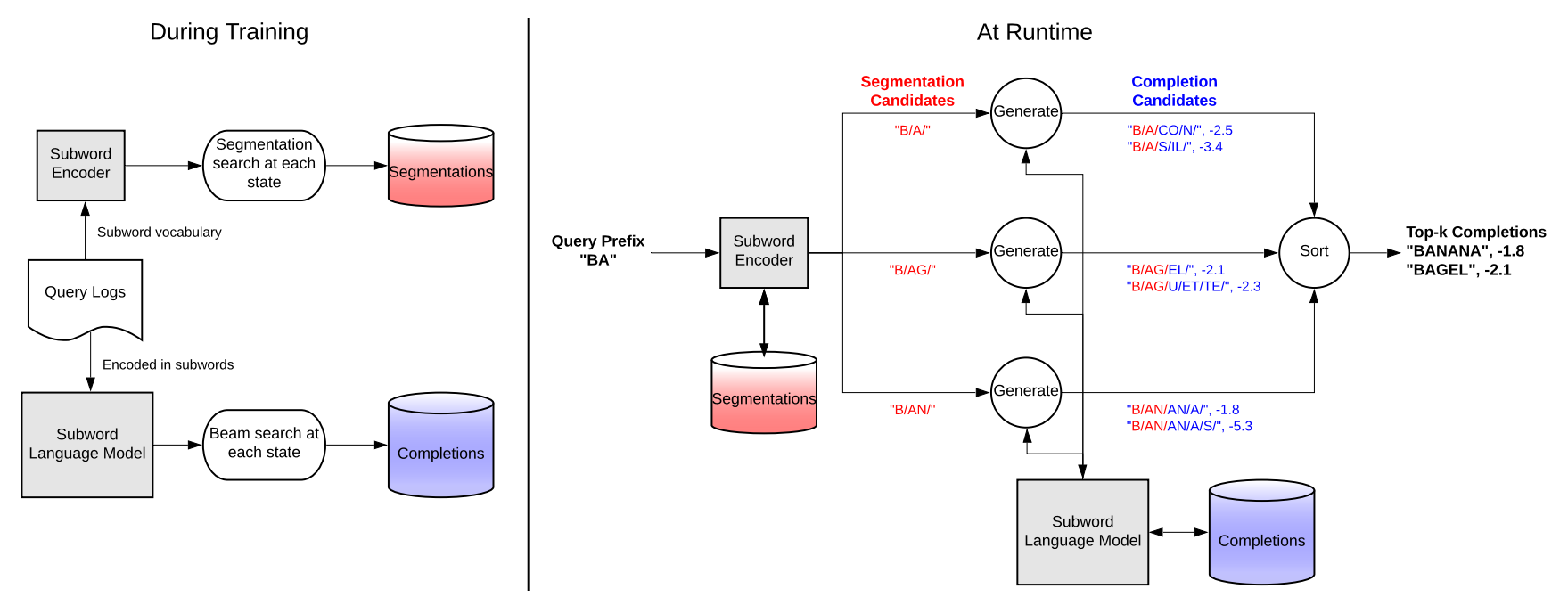

Figure 2: A flow chart for the proposed framework. During the training stage, an encoder and a language model are constructed. Segmentation search and beam search is performed, and the results are stored. At runtime, the encoder looks up segmentation candidates for the given input prefix and feeds them into the language model. The language model retrieves top- $k$ completions from each segmentation, all of which are merged into a single pool. Finally, the final top- $k$ completions from the pool are returned to the user.

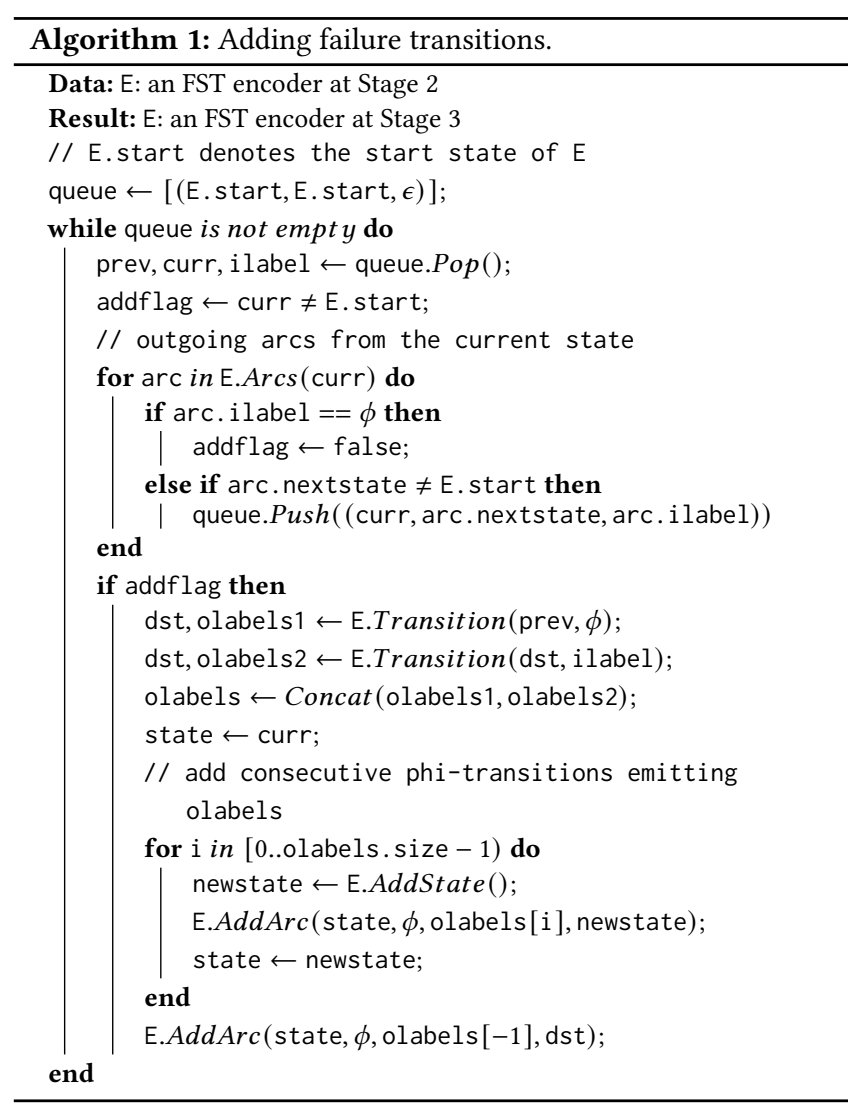

returns its final destination state and the output sequence collected along the way.

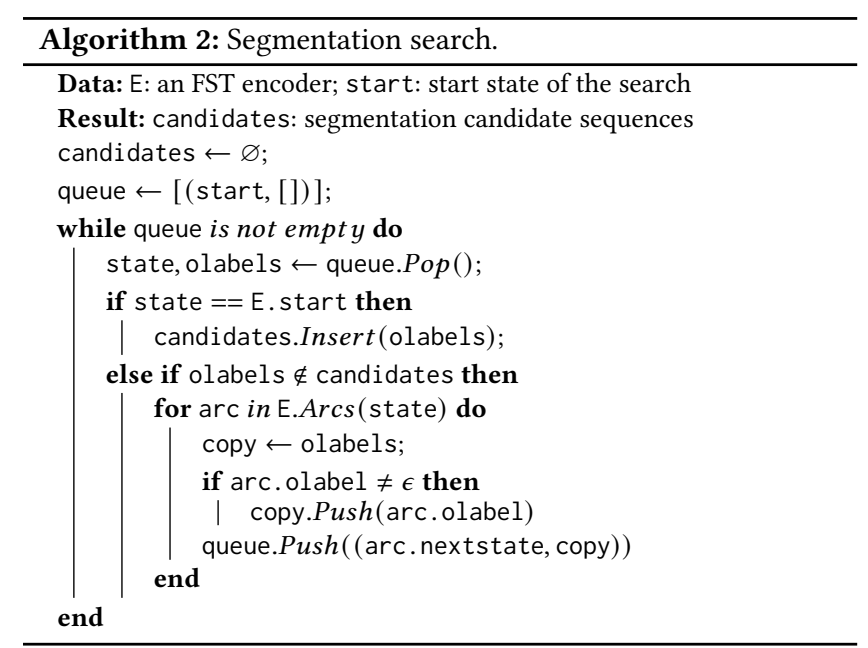

The FST in Figure 3c is an LPM encoder that transduces a sequence of input characters $\mathbf{x}$ to a subword sequence $\mathbf{y}$. Suppose a user input is $\mathbf{x}=$ "BA". The encoder goes through state transitions 05-0-1 and waits for further input from state 1 . When the user types additional characters, the encoder resumes making transitions from where it left off. Only when the user enters a newline character, the encoder makes exit transitions by following the $\phi$-arcs until reaching an exit state. For example, if the input is "BAN $\backslash n$ ", the complete path will be $0-5-0-1-2-7-0$, emitting the output sequence "B/A/N/".

4.1.2 Segmentation Search. The encoder E converts a complete query into its subword sequence, but it encounters the segmentation boundary problem [21] for a partial query prefix. For example, a prefix "BAN" may be the query "BAN" itself, to be encoded as 


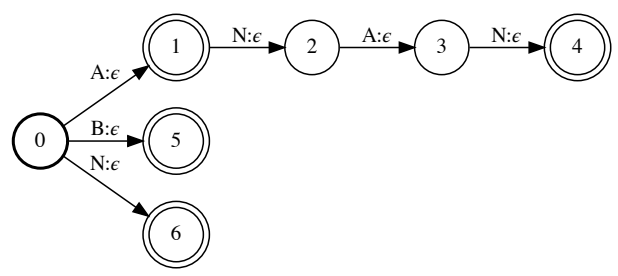

(a) Stage 1.

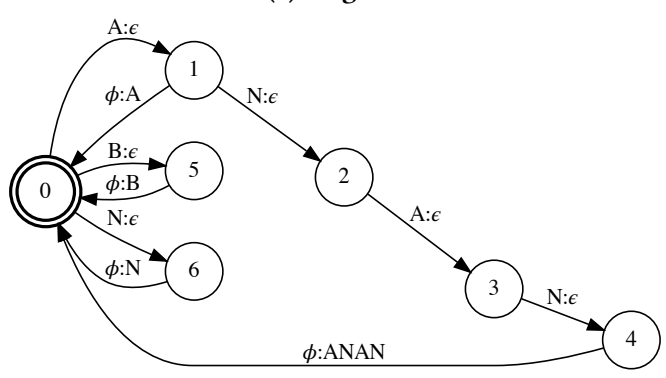

(b) Stage 2.

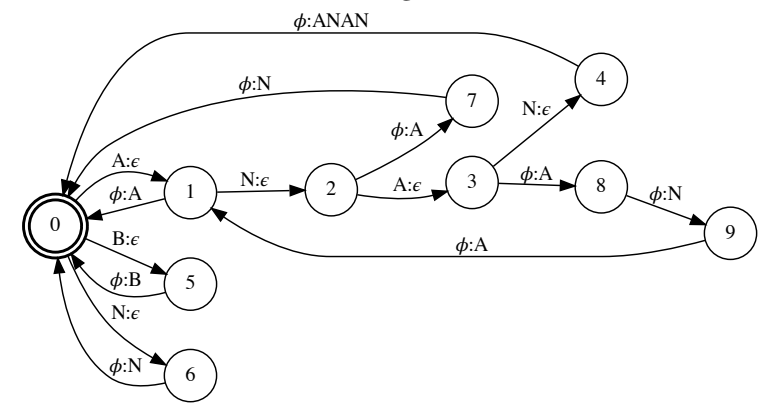

(c) Stage 3.

Figure 3: FST construction stages for a toy encoder example with the input character set $\mathcal{X}=\{\mathrm{A}, \mathrm{B}, \mathrm{N}\}$ and the output subword set $y=\{\mathrm{A}, \mathrm{B}, \mathrm{N}, \mathrm{ANAN}\}$.

"B/A/N/", but also can be a prefix to "BANANA", to be encoded as "B/ANAN/A/". The first token "B/" is determinate, but the second token is unknown and can be either "A/" or "ANAN/", depending on the future input. The system must consider every possible segmentation candidate during the beam search in order to provide accurate completions to the user. A straightforward algorithm to search for the segmentation candidates is to do a breadth-first traversal from the given state. During the search, we skip the path if its output sequence is already seen. We stop the search if the path reaches the start state of $E$. Algorithm 2 details the search process. Performing Algorithm 2 with start $=2$ results in candidates $=[" \mathrm{~A} / \mathrm{N} /$ ", "ANAN/"]. Concatenating each candidate with the determinate label "B/" yields two segmentation candidates " $\mathrm{B} / \mathrm{A} / \mathrm{N} /$ " and "B/ANAN/" as desired. Note that candidates is uniquely determined by the start state of the search. Hence, we perform the segmentation search prior to runtime by iterating the search from every state in the encoder. This effectively precomputes segmentation candidates for every possible input sequence.
Our segmentation search algorithm is similar to the retracing algorithm [21] in that it searches for all possible subword sequences. Unlike the retracing algorithm, however, the segmentation search does not need to retrace back to the very first character of the input; instead, it only retraces back to the first unknown character, reducing the number of candidates. Furthermore, the search is precomputed during the training stage, leading to higher efficiency. The space requirement for storing the segmentation candidates is negligible compared to that of the completions candidates.

\subsection{Language Model}

A language model estimates the probability distribution of a sequence of tokens $\mathbf{y}=\left(y_{1}, y_{2}, \ldots, y_{l}\right)$ as [20]

$$
p(\mathbf{y})=p\left(y_{1}, y_{2}, \ldots, y_{l}\right)=\prod_{j=1}^{l} p\left(y_{j} \mid y_{1}, y_{2}, \ldots, y_{j-1}\right) .
$$

A fully-generative QAC system [32] finds the best sequence of tokens with the highest probability among all sequences that start with the user's input prefix. The search is typically approximated by a beam search for efficiency.

4.2.1 N-Gram Model. In the classical n-gram language model, the conditional dependency is limited to a fixed number of tokens, thereby modeled as an $n$-th order Markov model. That is, the conditional probability of a token given a long history is approximated by limiting the history to the last $n-1$ tokens [20]

$$
p\left(y_{j} \mid y_{1}, y_{2}, \ldots, y_{j-1}\right) \approx p\left(y_{j} \mid y_{j-n+1}, y_{j-n+2}, \ldots, y_{j-1}\right) \text {. }
$$

A neural language model, on the other hand, can effectively model a longer sequence and has scored the state-of-the-art benchmark on numerous tasks [14, 28, 29, 41]. For this reason a neural language model has been employed in the recent generative approaches [21, 32, 42]. In our framework, however, we employ an $\mathrm{n}$-gram language model because its unique data structure enables generation of query completions prior to runtime. In particular, by limiting the context to a fixed number of previous tokens, we are able to precompute and store beam search results for every possible user input and still achieve better completion accuracy. Below we address two main concerns regarding an n-gram model.

Performance. An n-gram model may not be as effective as a neural model for a long sequence, but it is powerful when the sequence length is close to its order $n$. Most of the user queries entered in search engines are relatively short, consisting of only several words. As an illustrative example, the mean query length of the public AOL dataset [33] is 18 characters-long. This can be further reduced to less than 6 by encoding the queries with subword vocabulary of size 4096. In our experiment, we find that a 5-gram model consistently outperforms neural models [21,42] in completion accuracy by a large margin, evaluated on two different public datasets.

Space complexity. An n-gram model typically requires a larger memory footprint than a neural model because it stores probability distributions in the model. The large space complexity makes it challenging to deploy an n-gram model in systems with hard memory constraint, such as mobile devices. In typical large-scale servers, however, memory resources are cheap and abundant. In Section 5.4 
we report empirical studies on the trade-offs of completion accuracy and space complexity using an n-gram pruning technique.

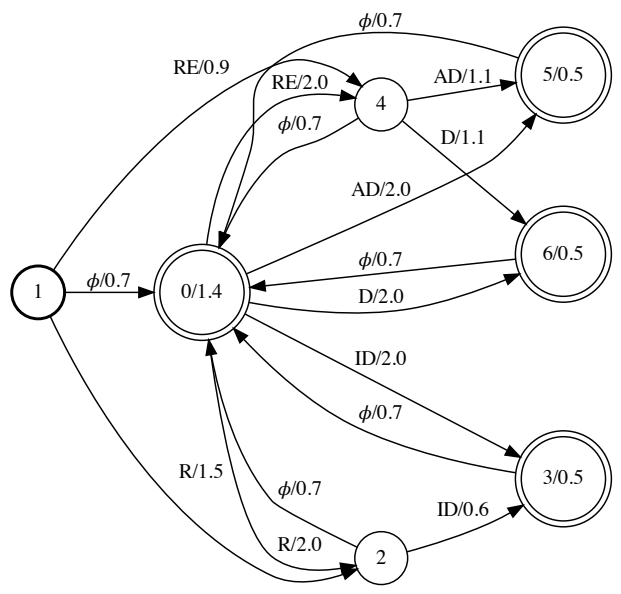

Figure 4: Weighted FST representation of a toy 2-gram subword language model. The output labels are omitted for simplicity. Each transition is marked by the input label $x$ and the weight $w$ as $x / w$, and each final state is marked with the index and the final weight.

4.2.2 Weighted FST Representation. Figure 4 illustrates a toy ngram language model represented as a weighted FST $G$ following [5, 35]. Each state in $G$ represents a history $h$, analogous to the hidden state vector in recurrent neural network (RNN) models. For example, the initial state 1 in $G$ represents the history of the start token " $\langle s\rangle$ ", and state 5 represents the history " $\langle\mathrm{s}\rangle / \mathrm{RE} / \mathrm{AD} /$ ". For simplicity, we assume that every input sequence begins with the start token and will not be written explicitly. Note that output labels in $G$ are omitted because they are always equal to the input labels ${ }^{5}$.

Each transition in the graph represents either the next symbol or a backoff, labeled by a $\phi$-symbol. The $\phi$-transition works the same way as defined in Section 4.1. A key observation is that for any input sequence of a finite length, the model makes state transitions of a finite length and lands on the state that is associated with the input sequence as its history. For example, an input sequence "RE/AD/" follows the state transitions 1-4-5, and hence is associated with the state 5 . Similarly, an input sequence "D/ID/" follows the state transitions 1-0-6-0-3 and is associated with the state 3 .

4.2.3 Beam Search. Because every possible input sequence of a finite length is associated with one of the states in the model, we iterate each state and generate top- $k$ completions via beam search during the training stage. We employ the standard beam search to generate most likely sequences conditioned on the current history or state. The process is very similar to [32] except that we use an $\mathrm{n}$-gram model at a subword-level. Essentially, a beam search with the beam width $\beta$ is a breadth-first search where at each level of the search tree, only the top- $\beta$ hypotheses are kept, reducing the search space. Running the beam search naively with an n-gram model is slow because the search has to loop through the conditional

\footnotetext{
${ }^{5}$ Here, a $\phi$-transition emits a blank label
}

probability $p(y \mid h)$ for every token $y \in \mathcal{Y}$. The larger the vocabulary size $|\mathcal{Y}|$, the slower the search runs. This differs from an RNN language model where one forward step returns the vector of the conditional probabilities for every token at once. A trick to expedite the search is to store a fixed number ${ }^{6}$ of top transitions for each state. Once the beam search is complete on all of the states, the data is no longer needed and can be removed. The space complexity for storing the completions is $O(Q k l)$ where $l$ is the average completion length, and $Q=|Q|$ is the number of the states.

The precomputation technique effectively stores the top- $k$ beam search results for every possible user input and differs from a caching technique that typically stores only a subset of the data. In a way, our method can be viewed as a caching with $100 \%$ hit-rate. We note that this technique is not feasible with neural language models because there are intractable number of states, as the effective number of states for an RNN model is $V^{n-1}$ where $V$ is the size of the vocabulary and $n$ is the upper limit in the n-gram order for caching. For comparison, our 4096-subword 8-gram model trained on the AOL dataset with moderate pruning has $Q \approx 9 \times 10^{6}$ states, taking up 7GB of space with precomputation, while an RNN model would require $4096^{7} \approx 2 \times 10^{25}$ effective states to store.

\section{EXPERIMENT}

\subsection{Dataset and Setup}

The AOL query logs [33] and the MSN data [46] are the two widely used public query dataset. However, we evaluate our system on the AOL query logs and the ORCAS dataset [13] as the MSN data "has not been released again since 2009" [13].

For the AOL logs, we closely follow $[6,21]$ where we normalize, remove duplicates from the same user, and filter out the queries with less than 3 characters. We time-split the queries and obtain the train set, validation set, and test set, each containing $17.5 \mathrm{M}$, $1.5 \mathrm{M}$, and $1.3 \mathrm{M}$ queries, respectively. Roughly a half of the test set is unseen in the training set. We then extract a test prefix $\mathbf{p}$ from each of the test query $\mathbf{q}$ by randomly selecting a prefix length $2 \leq l_{\mathrm{p}} \leq l_{\mathrm{q}}-1$, where $l_{\mathrm{p}}$ and $l_{\mathrm{q}}$ denote the prefix and query lengths, respectively. We ensure that prefixes of varying lengths are extracted from duplicate queries, simulating a more realistic scenario. For example, the query "google" appears more than 10,000 times in the test set, comprising $0.76 \%$ of the test data alone. We extract roughly equal numbers of prefixes "go", "goo", "goog", and "googl" from the test queries "google". This differs from $[6,21]$ where the prefix length is statically determined by the md5 hash value of the query, and only a single variant of prefix is tested for all of the duplicate queries.

We follow the same procedure with the ORCAS dataset and obtain the train, validation, and test sets, each containing $15 \mathrm{M}$, $1.9 \mathrm{M}$, and $1.9 \mathrm{M}$ queries, respectively. Here, we randomly split the data instead of splitting by time, as the query timestamps are not available. Roughly $40 \%$ of the test queries are unseen.

\subsection{Evaluation Metrics}

We consider two categories of performance metrics for the QAC system: 1) completion accuracy and 2) time and space complexity

\footnotetext{
${ }^{6}$ typically equal to the beam width.
} 
at runtime. Completion accuracy measures how well suggested completions match the user's intended query in a QAC system. Higher accuracy leads to better user experience, as the system helps the user search for the intended query with fewer keystrokes. We use two different metrics to quantify accuracy of the completions. The first is mean reciprocal rank (MRR) [11]. MRR for a test set $\mathcal{P}$ is evaluated as $\frac{1}{|\mathcal{P}|} \sum_{\mathbf{p} \in \mathcal{P}} \frac{1}{r_{\mathrm{p}}}$ where $|\mathcal{P}|$ is the number of the prefixes tested, $\mathbf{p}$ is a test prefix provided to the system, and $r_{\mathrm{p}}$ is the rank of the target query within the completion candidates. If the target query is not in the completion candidates, $r_{\mathrm{p}}$ is defined to be $\infty$.

The second accuracy metric used is success rate, which measures the ratio of the target query found within the completions. Success rate differs from MRR in that each completion is equally rewarded irrespective of its ranking and is always greater or equal to MRR.

Time and space complexity at runtime are the most critical metrics for large-scale deployments. We evaluate the time complexity with raw runtime completion speed, measured in queries per second (QPS). We also measure space complexity by reporting the system's total memory footprint in resident set size (RSS) including the precomputed results.

\subsection{Benchmark}

Table 1 summarizes our system's benchmark results and compares with the previous methods $[8,21,42]$ at $k=10$ and the beam width of 30 , evaluated on the AOL dataset [33]. We could not compare with [32] as the source code is not released. All of our evaluations were performed on an Intel Xeon Gold 5122 processor equipped with NVIDIA Quadro GV100. The best value in each column is marked in bold.

The first row is obtained from our own implementation of the traditional lookup-based approach [8]. We optimize for runtime by precomputing the top- $k$ completions associated with each node in the trie. This approach runs the fastest at 220,000 QPS. However, the overall accuracy is low due to its inability to suggest unseen queries. Note that this model is advantageous in speed over the rest of the systems because it terminates early and returns no candidates for the unseen queries.

The second row is obtained from Beam Search (LSTM-256) [42]. The overall accuracy is comparable to MPC but is slower by 1,000 times. The runtime we obtain (6ms) is twice faster than reported (13ms). A larger model is likely to improve the accuracy but at the expense of slower runtime: [42] reports that LSTM-1024 is about 10 times slower than LSTM-256.

Rows 3-5 are obtained from the char and SR models in [21] running on the GPU. The MRR results are different from those reported in [21] due to differences in test prefix extraction discussed earlier. We ran the test with a batch size of 64 to take full advantage of the GPU hardware, expediting the runtime speed by six times than reported in [21]. The SR-4096 model (row 5) runs the fastest at 210 QPS among the generative systems employing neural models.

Finally, the last three rows are obtained with our models. We train three different models: an 8-gram model at the character-level (QBZchar) and two 5-gram subword models with the vocabulary size 256 and 4096 (QBZ-256 and QBZ-4096). We use sentencepiece [24] to extract subword vocabulary using the BPE method. We construct LPM encoders, train the n-gram models with KenLM $[17,18]$ and convert to the OpenFST $[4,7]$ format using the OpenGRM NGram library $[5,35]$. We precompute both of the segmentation and completions candidates and store them in memory. While it is also possible to implement the language model directly on the KenLM's own optimized trie data structure, we use the general-purpose FST library because we want to illustrate the high-level architecture of the system rather than a specific implementation or optimization.

QBZ-4096 consistently achieves the best accuracy, in MRR and success rate, outperforming the MPC model even for the seen queries. At the same time, it also achieves roughly 100x faster speed compared to the previous generative systems. Comparing it with QBZ-256, we observe that larger vocabulary size results in higher accuracy-because it can model longer context-but at slower speed-because there are more segmentation candidates to consider. QBZ-char achieves the fastest runtime among the three but is not as effective, as it can only account for up to seven previous characters.

Note that the training time includes language model training as well as precomputation. ${ }^{7}$ The training time for the neural language model is the major bottleneck in the previous generative methods. To reduce the training time in our system, we implement parallel precomputation where the beam search is run independently for each state on 16 threads concurrently. Shorter training time is advantageous in QAC systems as it allows more frequent model updates that can accommodate the most recent data.

To validate our system further, we repeat the same experiment on the ORCAS dataset [13] and report in Table 2. We observe the similar trend in that QBZ-4096 performs the best accuracy score among all the models tested. The training time and runtime speed are comparable to Table 1 and not reported.

\subsection{Model Pruning}

Next, we investigate a trade-off between accuracy and memory footprint with various pruning configurations. For simplicity, we explore only the most straightforward pruning method: count cutoffs [39] where n-grams with count less or equal to the cutoff is removed. For example, count cutoff 123 denotes removing unigrams of count less or equal to 1 , bigrams of count less or equal to 2, and trigrams and higher-order n-grams of count less or equal to 3 . Table 3 summarizes the system's overall accuracy and total memory footprint for models of different orders and count cutoffs. The first three models are identical to the QBZ models in Table 1. QBZ-4096 is not pruned and requires the largest memory at $29.8 \mathrm{~GB}$ with the highest number of states $Q$. The system's space complexity is roughly $O(Q)$, as the language model and the completion results account for the majority of the memory. We find that the number of states does not necessarily correlate with completion accuracy. For example, the 8-gram models of vocabulary size 256 with moderate pruning (rows 4 and 5) have fewer states than QBZ-256 but achieve higher accuracy.

On the extreme end is the 5-gram model of vocabulary size 4096 with an aggressive pruning (the last row), measuring only $400 \mathrm{MB}$ in memory footprint. This model achieves comparable accuracy to QBZ-char with less than one-tenth of the memory space. The subword vocabulary size, and the n-gram order, and its pruning

\footnotetext{
${ }^{7}$ applicable to MPC and QBZ models.
} 
Table 1: QAC system benchmark results with the proposed framework evaluated on the AOL dataset [33]. We compare our systems with the traditional lookup-based method and the previous generative systems based on neural models.

\begin{tabular}{c|c|c|ccc|ccc|cc|c}
\hline & & & \multicolumn{4}{|c|}{ MRR } & \multicolumn{3}{|c}{ Success Rate } & Speed & Runtime Hardware \\
Model & Vocab Size & Training Time & Seen & Unseen & All & Seen & Unseen & All & (QPS) & (\#Treads) \\
\hline MPC [8] & char & 5 mins & 0.606 & 0.000 & 0.308 & 0.761 & 0.000 & 0.388 & $\mathbf{2 2 0 , 0 0 0}$ & CPU (1) \\
\hline Wang [42] & char & 8 hours & 0.451 & 0.180 & 0.318 & 0.540 & 0.228 & 0.387 & 160 & CPU (8) \\
\hline \multirow{3}{*}{ Kim [21] } & char & & 0.390 & 0.159 & 0.272 & 0.507 & 0.234 & 0.367 & 60 & \\
& 256 & \multirow{3}{*}{$<$ hours } & 0.368 & 0.139 & 0.255 & 0.475 & 0.193 & 0.337 & 120 & GPU \\
& 4096 & & 0.357 & 0.134 & 0.247 & 0.468 & 0.189 & 0.331 & 210 & \\
\hline QBZ-char & char & & 0.540 & 0.203 & 0.374 & 0.698 & 0.278 & 0.492 & 73,000 & \multirow{2}{*}{ CPU (1) } \\
QBZ-256 & 256 & \multirow{2}{*}{$<15$ mins } & 0.562 & 0.210 & 0.389 & 0.723 & 0.290 & 0.510 & 50,000 & \\
QBZ-4096 & 4096 & & $\mathbf{0 . 6 1 2}$ & $\mathbf{0 . 2 1 7}$ & $\mathbf{0 . 4 1 8}$ & $\mathbf{0 . 7 6 6}$ & $\mathbf{0 . 2 9 9}$ & $\mathbf{0 . 5 3 7}$ & 14,000 & \\
\hline
\end{tabular}

Table 2: QAC system benchmark evaluated on the ORCAS [13] dataset.

\begin{tabular}{c|ccc|ccc}
\hline \multirow{2}{*}{ Model } & \multicolumn{3}{|c|}{ MRR } & \multicolumn{3}{c}{ Success Rate } \\
& Seen & Unseen & All & Seen & Unseen & All \\
\hline MPC & 0.398 & 0.000 & 0.242 & 0.563 & 0.000 & 0.342 \\
\hline Wang & 0.234 & 0.199 & 0.220 & 0.288 & 0.248 & 0.273 \\
\hline Kim-char & 0.210 & 0.165 & 0.192 & 0.293 & 0.232 & 0.269 \\
Kim-256 & 0.188 & 0.151 & 0.173 & 0.263 & 0.214 & 0.244 \\
Kim-4096 & 0.183 & 0.146 & 0.169 & 0.263 & 0.211 & 0.242 \\
\hline QBZ-char & 0.290 & 0.232 & 0.267 & 0.399 & 0.315 & 0.366 \\
QBZ-256 & 0.334 & 0.248 & 0.300 & 0.461 & 0.341 & 0.414 \\
QBZ-4096 & $\mathbf{0 . 4 1 4}$ & $\mathbf{0 . 2 5 8}$ & $\mathbf{0 . 3 5 3}$ & $\mathbf{0 . 5 6 5}$ & $\mathbf{0 . 3 6 2}$ & $\mathbf{0 . 4 8 5}$ \\
\hline
\end{tabular}

option can serve as attributes to adopt in a system by weighing the trade-offs among accuracy, memory requirement, and runtime speed.

\section{CONCLUSION}

We present a scalable query completion framework with excellent accuracy by generating likely completions from a statistical language model. We introduce several techniques to shift computational overhead to pre-runtime and demonstrate n-gram pruning as an effective option for systems with constrained memory. The research question we address is whether we can design a generative system that is an attractive choice for large deployments. Experimental results on two different public datasets consistently show that our system 1) is as capable as the traditional MPC system for the seen queries, 2) is able to suggest completions for tail queries with great accuracy, and 3) is 100x faster than previous generative systems.

While our results are very promising on the data we tested, an investigation on the completion accuracy with longer queries would corroborate our experiments and is left as future work. Another direction in extending our work is to hybridize the framework with the traditional approach to further narrow the gap in efficiency.

\section{REFERENCES}

[1] [n.d.]. Bert Tokenizer Source Code. https://github.com/google-research/bert/ blob/eedf5716ce1268e56f0a50264a88cafad334ac61/tokenization.py\#L311. Accessed: 2020-06-29.
[2] [n.d.]. Google Search scratches its brain 500 million times a day. https://www. cnet.com/news/google-search-scratches-its-brain-500-million-times-a-day/. Accessed: 2020-07-14.

[3] [n.d.]. How Google autocomplete works in Search. https://www.blog.google/ products/search/how-google-autocomplete-works-search/. Accessed: 2020-0625.

[4] [n.d.]. OpenFst Library. http://www.openfst.org/twiki/bin/view/FST/WebHome. Accessed: 2020-07-27.

[5] [n.d.]. OpenGRM NGram Library. http://www.openfst.org/twiki/bin/view/GRM/ NGramLibrary. Accessed: 2020-07-01.

[6] [n.d.]. Subword Language Model for Query Auto-Completion. https://github. com/clovaai/subword-qac. Accessed: 2020-07-01.

[7] Cyril Allauzen, Michael Riley, Johan Schalkwyk, Wojciech Skut, and Mehryar Mohri. 2007. OpenFst: A General and Efficient Weighted Finite-State Transducer Library. In CIAA.

[8] Ziv Bar-Yossef and Naama Kraus. 2011. Context-Sensitive Query AutoCompletion. In Proceedings of the 20th International Conference on World Wide Web (Hyderabad, India) (WWW'11). ACM, New York, NY, USA, 107-116.

[9] Sumit Bhatia, Debapriyo Majumdar, and Prasenjit Mitra. 2011. Query Suggestions in the Absence of Query Logs. In Proceedings of the 34th International ACM SIGIR Conference on Research and Development in Information Retrieval (Beijing, China) (SIGIR '11). Association for Computing Machinery, New York, NY, USA, 795-804.

[10] Fei Cai and Maarten de Rijke. 2016. A Survey of Query Auto Completion in Information Retrieval. Now Publishers Inc., Hanover, MA, USA.

[11] Jaime Carbonell and Jade Goldstein. 1998. The Use of MMR, Diversity-Based Reranking for Reordering Documents and Producing Summaries. In Proceedings of the 21st Annual International ACM SIGIR Conference on Research and Development in Information Retrieval (Melbourne, Australia) (SIGIR '98). Association for Computing Machinery, New York, NY, USA, 335-336.

[12] Kenneth Church, Bo Thiesson, and Robert Ragno. 2007. K-Best Suffix Arrays. In Human Language Technologies 2007: The Conference of the North American Chapter of the Association for Computational Linguistics; Companion Volume, Short Papers. Association for Computational Linguistics, Rochester, New York, 17-20.

[13] Nick Craswell, Daniel Campos, Bhaskar Mitra, Emine Yilmaz, and Bodo Billerbeck. 2020. ORCAS: 20 Million Clicked Query-Document Pairs for Analyzing Search. In Proceedings of the 29th ACM International Conference on Information \& Knowledge Management (Virtual Event, Ireland) (CIKM '20). Association for Computing Machinery, New York, NY, USA, 2983-2989.

[14] Zihang Dai, Zhilin Yang, Yiming Yang, Jaime Carbonell, Quoc Le, and Ruslan Salakhutdinov. 2019. Transformer-XL: Attentive Language Models beyond a Fixed-Length Context. In Proceedings of the 57th Annual Meeting of the Association for Computational Linguistics. Association for Computational Linguistics, Florence, Italy, 2978-2988.

[15] Jacob Devlin, Ming-Wei Chang, Kenton Lee, and Kristina Toutanova. 2019. BERT: Pre-training of Deep Bidirectional Transformers for Language Understanding. In Proceedings of the 2019 Conference of the North American Chapter of the Association for Computational Linguistics: Human Language Technologies, Volume 1 (Long and Short Papers). Association for Computational Linguistics, Minneapolis, Minnesota, 4171-4186.

[16] J. Drexler and J. Glass. 2020. Learning a Subword Inventory Jointly with Endto-End Automatic Speech Recognition. In ICASSP 2020 - 2020 IEEE International Conference on Acoustics, Speech and Signal Processing (ICASSP). 6439-6443.

[17] Kenneth Heafield. 2011. KenLM: Faster and Smaller Language Model Queries. In Proceedings of the Sixth Workshop on Statistical Machine Translation. Association for Computational Linguistics, Edinburgh, Scotland, 187-197.

[18] Kenneth Heafield, Ivan Pouzyrevsky, Jonathan H. Clark, and Philipp Koehn. 2013. Scalable Modified Kneser-Ney Language Model Estimation. In Proceedings of the 
Table 3: Comparison of completion accuracy and memory footprint for various pruning configurations tested on the AOL dataset [33]. The memory footprint denotes the total memory requirement for the system, including storage for the precomputed results.

\begin{tabular}{c|c|c|c|c|c|c|c}
\hline Model & Vocab Size & n-gram Order & Count Cutoff & \# States & MRR & Success Rate & Memory Footprint \\
\hline QBZ-char & char & 8 & 011222334 & $6.6 \mathrm{M}$ & 0.374 & 0.492 & $5.4 \mathrm{~GB}$ \\
QBZ-256 & 256 & 5 & 0000 & $14.5 \mathrm{M}$ & 0.389 & 0.510 & $11.1 \mathrm{~GB}$ \\
QBZ-4096 & 4096 & 5 & 00000 & $40.6 \mathrm{M}$ & $\mathbf{0 . 4 1 8}$ & $\mathbf{0 . 5 3 7}$ & $29.8 \mathrm{~GB}$ \\
\hline- & 256 & 8 & 01122334 & $12.4 \mathrm{M}$ & 0.409 & 0.525 & $9.4 \mathrm{~GB}$ \\
- & 256 & 8 & 12345678 & $5.9 \mathrm{M}$ & 0.401 & 0.516 & $4.5 \mathrm{~GB}$ \\
- & 256 & 5 & 13579 & $1.5 \mathrm{M}$ & 0.379 & 0.496 & $1.2 \mathrm{~GB}$ \\
- & 256 & 5 & 111213141 & $0.5 \mathrm{M}$ & 0.362 & 0.473 & $\mathbf{0 . 4} \mathrm{GB}$ \\
\hline- & 4096 & 8 & 01122334 & $9.2 \mathrm{M}$ & 0.413 & 0.528 & $6.8 \mathrm{~GB}$ \\
- & 4096 & 8 & 12345678 & $3.8 \mathrm{M}$ & 0.406 & 0.519 & $2.9 \mathrm{~GB}$ \\
- & 4096 & 5 & 13579 & $1.7 \mathrm{M}$ & 0.399 & 0.512 & $1.3 \mathrm{~GB}$ \\
- & 4096 & 5 & 111213141 & $0.4 \mathrm{M}$ & 0.378 & 0.485 & $\mathbf{0 . 4} \mathbf{G B}$ \\
\hline
\end{tabular}

51st Annual Meeting of the Association for Computational Linguistics (Volume 2: Short Papers). Association for Computational Linguistics, Sofia, Bulgaria, 690696.

[19] Bo-June (Paul) Hsu and Giuseppe Ottaviano. 2013. Space-Efficient Data Structures for Top-k Completion. In Proceedings of the 22nd International Conference on World Wide Web (Rio de Janeiro, Brazil) (WWW'13). Association for Computing Machinery, New York, NY, USA, 583-594.

[20] Daniel Jurafsky and James H. Martin. 2000. Speech and Language Processing: An Introduction to Natural Language Processing, Computational Linguistics, and Speech Recognition (1st ed.). Prentice Hall PTR, USA.

[21] Gyuwan Kim. 2019. Subword Language Model for Query Auto-Completion. In EMNLP/IFCNLP.

[22] Unni Krishnan, Alistair Moffat, and Justin Zobel. 2017. A Taxonomy of Query Auto Completion Modes. In Proceedings of the 22nd Australasian Document Computing Symposium (Brisbane, QLD, Australia) (ADCS 2017). Association for Computing Machinery, New York, NY, USA, Article 6, 8 pages.

[23] Taku Kudo. 2018. Subword Regularization: Improving Neural Network Translation Models with Multiple Subword Candidates. ArXiv abs/1804.10959 (2018).

[24] Taku Kudo and John Richardson. 2018. SentencePiece: A simple and language independent subword tokenizer and detokenizer for Neural Text Processing. In Proceedings of the 2018 Conference on Empirical Methods in Natural Language Processing: System Demonstrations. Association for Computational Linguistics, Brussels, Belgium, 66-71.

[25] Werner Kuich and Arto Salomaa (Eds.). 1985. Semirings, Automata, Languages. Springer-Verlag, Berlin, Heidelberg.

[26] Guoliang Li, Shengyue Ji, Chen Li, and Jianhua Feng. 2009. Efficient Type-Ahead Search on Relational Data: A TASTIER Approach. In Proceedings of the 2009 ACM SIGMOD International Conference on Management of Data (Providence, Rhode Island, USA) (SIGMOD '09). Association for Computing Machinery, New York, NY, USA, 695-706.

[27] David Maxwell, Peter Bailey, and David Hawking. 2017. Large-Scale Generative Query Autocompletion. In Proceedings of the 22nd Australasian Document Computing Symposium (Brisbane, QLD, Australia) (ADCS 2017). Association for Computing Machinery, New York, NY, USA, Article 9, 8 pages.

[28] Bryan McCann, Nitish Shirish Keskar, Caiming Xiong, and Richard Socher. 2018 The Natural Language Decathlon: Multitask Learning as Question Answering. arXiv preprint arXiv:1806.08730 (2018).

[29] Tomas Mikolov, Martin Karafiát, Lukas Burget, Jan Cernocký, and Sanjeev Khudanpur. 2010. Recurrent neural network based language model. Proceedings of the 11th Annual Conference of the International Speech Communication Association, INTERSPEECH 2010 2, 1045-1048.

[30] Bhaskar Mitra and Nick Craswell. 2015. Query Auto-Completion for Rare Prefixes. In Proceedings of the 24th ACM International on Conference on Information and Knowledge Management (Melbourne, Australia) (CIKM '15). Association for Computing Machinery, New York, NY, USA, 1755-1758.

[31] Mehryar Mohri, Fernando Pereira, and Michael Riley. 2002. Weighted FiniteState Transducers in Speech Recognition. Comput. Speech Lang. 16, 1 (Jan. 2002), 69-88.

[32] Dae Hoon Park and Rikio Chiba. 2017. A Neural Language Model for Query Auto-Completion. Proceedings of the 40th International ACM SIGIR Conference on Research and Development in Information Retrieval (2017).

[33] Greg Pass, Abdur Chowdhury, and Cayley Torgeson. 2006. A Picture of Search. In Proceedings of the 1st International Conference on Scalable Information Systems (Hong Kong) (InfoScale '06). Association for Computing Machinery, New York,
NY, USA, 1-es

[34] Alec Radford, Jeffrey Wu, Rewon Child, David Luan, Dario Amodei, and Ilya Sutskever. 2018. Language Models are Unsupervised Multitask Learners. (2018). https://d4mucfpksywv.cloudfront.net/better-language-models/languagemodels.pdf

[35] Brian Roark, Richard Sproat, Cyril Allauzen, Michael Riley, Jeffrey Sorensen, and Terry Tai. 2012. The OpenGrm open-source finite-state grammar software libraries. In Proceedings of the ACL 2012 System Demonstrations. Association for Computational Linguistics, Jeju Island, Korea, 61-66.

[36] M. Schuster and K. Nakajima. 2012. Japanese and Korean voice search. In 2012 IEEE International Conference on Acoustics, Speech and Signal Processing (ICASSP). 5149-5152.

[37] Rico Sennrich, Barry Haddow, and Alexandra Birch. 2016. Neural Machine Translation of Rare Words with Subword Units. In Proceedings of the 54th Annual Meeting of the Association for Computational Linguistics (Volume 1: Long Papers). Association for Computational Linguistics, Berlin, Germany, 1715-1725.

[38] Yelong Shen, Xiaodong He, Jianfeng Gao, Li Deng, and Grégoire Mesnil. 2014. Learning Semantic Representations Using Convolutional Neural Networks for Web Search. In Proceedings of the 23rd International Conference on World Wide Web (Seoul, Korea) (WWW'14 Companion). Association for Computing Machinery, New York, NY, USA, 373-374.

[39] V. Siivola, T. Hirsimaki, and S. Virpioja. 2007. On Growing and Pruning Kneser-Ney Smoothed N-Gram Models. IEEE Transactions on Audio, Speech, and Language Processing 15, 5 (2007), 1617-1624.

[40] Idan Szpektor, Aristides Gionis, and Yoelle Maarek. 2011. Improving Recommendation for Long-Tail Queries via Templates. In Proceedings of the 20th International Conference on World Wide Web (Hyderabad, India) (WWW'11). Association for Computing Machinery, New York, NY, USA, 47-56.

[41] Ashish Vaswani, Noam Shazeer, Niki Parmar, Jakob Uszkoreit, Llion Jones, Aidan N Gomez, Ł ukasz Kaiser, and Illia Polosukhin. 2017. Attention is All you Need. In Advances in Neural Information Processing Systems 30, I. Guyon, U. V. Luxburg, S. Bengio, H. Wallach, R. Fergus, S. Vishwanathan, and R. Garnett (Eds.). Curran Associates, Inc., 5998-6008.

[42] Po-Wei Wang, Huan Zhang, Vijai Mohan, Inderjit S. Dhillon, and J. Zico Kolter. 2018. Realtime Query Completion via Deep Language Models. In eCOM@SIGIR.

[43] Weiran Wang, Guangsen Wang, Aadyot Bhatnagar, Yingbo Zhou, Caiming Xiong, and Richard Socher. 2020. An investigation of phone-based subword units for end-to-end speech recognition. arXiv:2004.04290 [eess.AS]

[44] Yonghui Wu, Mike Schuster, Zhifeng Chen, Quoc V. Le, Mohammad Norouzi, Wolfgang Macherey, Maxim Krikun, Yuan Cao, Qin Gao, Klaus Macherey, Jeff Klingner, Apurva Shah, Melvin Johnson, Xiaobing Liu, Lukasz Kaiser, Stephan Gouws, Yoshikiyo Kato, Taku Kudo, Hideto Kazawa, Keith Stevens, George Kurian, Nishant Patil, Wei Wang, Cliff Young, Jason Smith, Jason Riesa, Alex Rudnick, Oriol Vinyals, Gregory S. Corrado, Macduff Hughes, and Jeffrey Dean. 2016. Google's Neural Machine Translation System: Bridging the Gap between Human and Machine Translation. ArXiv abs/1609.08144 (2016).

[45] Aston Zhang, Amit Goyal, Weize Kong, Hongbo Deng, Anlei Dong, Yi Chang, Carl A. Gunter, and Jiawei Han. 2015. AdaQAC: Adaptive Query Auto-Completion via Implicit Negative Feedback. In Proceedings of the 38th International ACM SIGIR Conference on Research and Development in Information Retrieval (Santiago, Chile) (SIGIR '15). Association for Computing Machinery, New York, NY, USA, 143-152.

[46] Yuye Zhang and Alistair Moffat. 2006. Some Observations on User Search Behavior. Austr. 7. Intelligent Information Processing Systems. 